\title{
FeNO for detecting lower airway involvement in patients with allergic rhinitis
}

\author{
ZHENG ZHU, YANQING XIE, WEIJIE GUAN, YI GAO, SHU XIA, NANSHAN ZHONG and JINPING ZHENG \\ State Key Laboratory of Respiratory Disease, China Clinical Research Center of Respiratory Disease, \\ First Affiliated Hospital of Guangzhou Medical University, Guangzhou, Guangdong 510120, P.R. China
}

Received June 16, 2015; Accepted July 19, 2016

DOI: $10.3892 /$ etm.2016.3616

\begin{abstract}
Allergic rhinitis (AR) is a risk factor for asthma development. The value of fractional exhaled nitric oxide (FeNO) in detecting lower airway involvement in the progress of AR-asthma march has not been evaluated. The aim of the present study was to investigate the value of FeNO in assessing lower airway inflammation and predicting bronchial hyperresponsiveness (BHR) in AR with or without asthma. FeNO and eosinophil count in induced sputum, and a methacholine bronchial provocation test were performed in 93 subjects, including: 45 AR patients (AR group); 20 patients with AR and asthma (AR with asthma group); and 28 normal controls (control group). The AR group was divided into two sub-groups: AR with asymptomatic BHR group and AR without BHR group. Correlation between FeNO and eosinophil count was assessed. Receiver operating characteristic (ROC) curve was applied to evaluate the predictive and diagnostic value of FeNO in detecting BHR. The values of FeNO in the AR and AR with asthma groups were higher [29.5 (22.0) ppb and 61.5 (33.0) ppb] compared with the normal control group (16.0 (10.0) ppb), where the values in brackets indicate the interquartile range of the values. The percentages of eosinophils in induced sputum were $2.43 \pm 3.56,7.36 \pm 4.98$ and $18.58 \pm 11.26 \%$ in the control, AR and AR with asthma groups, respectively. For the diagnosis of BHR, the area under the curve (AUC) was 0.910 (95\% CI 0.836, 0.984), with the sensitivity and specificity 0.846 and 0.817 when the cut-off value takes $31.5 \mathrm{ppb}$. For diagnosis of asthma, the AUC was $0.873(95 \% \mathrm{CI}$ $0.753,0.992$ ) with sensitivity 0.857 and specificity 0.847 when taking the cut-off value to be $38.0 \mathrm{ppb}$. The value of FeNO was well correlated with eosinophil count in the sputum. The
\end{abstract}

Correspondence to: Professor Jinping Zheng, State Key Laboratory of Respiratory Disease, China Clinical Research Center of Respiratory Disease, First Affiliated Hospital of Guangzhou Medical University, 151 Yanjiang Road, Guangzhou, Guangdong 510120, P.R. China

E-mail: jpzhenggy@163.com

Key words: fractional exhaled nitric oxide, allergic rhinitis, bronchial hyperresponsiveness, asthma, induced sputum measurement of FeNO is an effective method in detecting lower airway involvement in AR developing to asthma.

\section{Introduction}

Allergic rhinitis (AR) and asthma are common diseases with an increasing prevalence, which, seriously impact the quality of life of patients (1). A proportion of AR patients develop asymptomatic bronchial hyperresponsiveness (BHR), and finally exhibit lower airway symptoms including coughing, wheezing and breath tightness, which may develop into asthma. Since is has been reported that AR and asthma are associated 'one airway, one disease' (2), an increasing number of studies have focused on the association between these conditions. The epidemiology, pathophysiology, anatomy, immunology evidences, the consistent response of upper and lower airways to the treatment of steroids (3-5). Furthermore, international guidelines (ARIA) (6) have confirmed the association and impact of them on each other.

Patients suffering from AR without asthma may present with elevated lowerairway inflammation (7). A number of mechanisms have been proposed to underlie the association between AR and asthma. One of the these suggests that the inflammation extends from upper airway to lower airways $(8,9)$. Various methods exist for the assessment of airway inflammations, including biopsy, lavage and cytology tests (10). These approaches offer sensitivity, but may be invasive or time-consuming and require professional or technical operation (11).

The measurement of fractional exhaled nitric oxide (FeNO) is a simple, safe and noninvasive method to detect airway inflammation, which is also correlated well with eosinophil count and eosinophil cationic protein in induced sputum or lavage fluid $(12,13)$. FeNO is a widely used metric for the evaluation and management of airway inflammatory diseases, such as asthma; however, the measurement of FeNO as a predictor for the diagnosis of BHR or asthma in AR patients has not been well investigated. The objective of the present study was to evaluate the measurement of FeNO for predicting BHR in AR patients asymptomatic for asthma and patients with AR combined with asthma.

\section{Materials and methods}

Subjects. A total of 102 patients with AR (including AR with asymptomatic BHR), normal controls (atopic or non-atopic) and 
AR combined with asthma (AR with asthma) that consulted the out-patient department in the First Affiliated Hospital of Guangzhou Medical University (Guangzhou, China) were recruited between September 2012 and April 2014 (Fig. 1). After screening, the subjects were divided into the AR group, AR with asthma group and normal control group. The AR group was then divided into two sub-groups: AR with asymptomatic BHR group (AR with BHR) and AR without BHR group. The diagnosis of AR and asthma were based on the guidelines of ARIA 2010 and GINA 2011 respectively $(6,14)$. Inclusion criteria: AR patients (male or female) and AR combined with asthma aged 18-50 years old, with a positive skin prick test for at least one type of aerogel allergen. The diagnoses of asthma were required to be $>3$ months and the patients were not regularly receiving medication. Exclusion criteria: Patients with exacerbation of AR or asthma within four weeks; with other respiratory diseases (e.g. chronic obstructive pulmonary disease); under the treatment of immunotherapy, leukotriene receptor antagonist, antihistamines, oral/inhaled corticosteroids or other medicines that may affect the results of the study within four weeks prior to the study; pregnant or breast-feeding women.

Study design. The study protocol was registered on https://clinicaltrials.gov/ (NCT01963741) and was approved by the Ethics Committee of The First Affiliated Hospital of Guangzhou Medical University (approval no. 2012-18). Written informed consent was obtained from each participant prior to the study.

As shown in Fig. 1, following screening, all enrolled patients underwent measurement of FeNO, methacholine (Provocholine, Methapharm Inc., Brantford, ON, Canada) bronchial provocation test (Mch-BPT) and induced sputum. Eosinophil count in induced sputum was conducted by routing method (15).

\section{Measurements}

FeNO. The measurement of FeNO was performed by using a NIOX MINO ${ }^{\circledR}$ medical device (Aerocrine AB, Solna, Sweden). All procedures met the recommendations of the American Thoracic Society and the European Respiratory Society (ATS/ERS) on FeNO measurement (16). The normal value of FeNO in Guangzhou was 17.7 (6.1) ppb, 95\% CI (6.1-41.0 ppb) (17).

Mch-BPT. Spirometry and Mch-BPT were performed using a spirometer (Jaeger Masterscreen) and automated APS pro system (CareFusion; BD Biosciences, San Diego, CA, USA), which meet the joint recommendations of the ATS/ERS (18). At least three maneuvers $(\leq 8)$ were required with the variation of forced vital capacity (FVC), and forced expiratory volume in $1 \mathrm{sec}\left(\mathrm{FEV}_{1}\right)$ between the best value best two values $<5 \%$ or $150 \mathrm{ml}$. FVC and $\mathrm{FEV}_{1}$ reported the best records. The predicted values were calculated based on the equation of Zheng and Zhong (19). The provocation was performed in a stepwise manner with initial and ultimate cumulative doses 0.390 and $12.520 \mathrm{mmol}$, respectively.

Sputum induction. Sputum was induced by using 3-4\% hypertonic saline nebulized for 10-20 min. Patients with

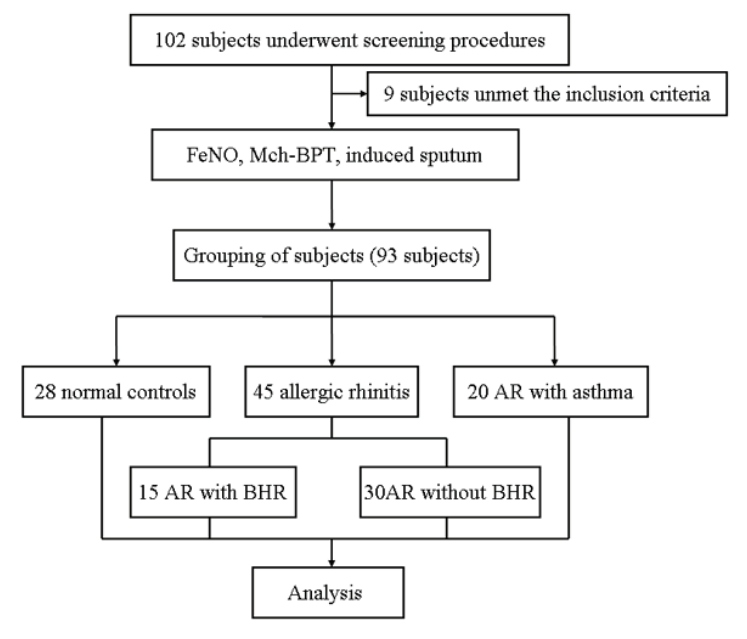

Figure 1. Patient profiles. FeNO, fractional exhaled nitric oxide; Mch-BPT, methacholine bronchial provocation test AR, allergic rhinitis; BHR, bronchial hyperresponsiveness.

BHR inhaled $200 \mu \mathrm{g}$ salbutamol through a spacer prior to the hypertonic saline nebulization. The induced sputum was collected, and the inflammation cells viability was identified by trypan blue; the sample was considered acceptable when the viability of the inflammation cells in the sputum was $>50 \%$ and the ratio of epithelial cells $<20 \%$ (15). A total of 400 non-epithelial cells were counted under 200 times scope of the microscope with HE staining, the percentages of eosinophils were reported.

Statistical analysis. Statistical analysis was performed with the SPSS 16.0 version package (SPSS, Inc., Chicago, IL, USA). Data were expressed as mean \pm standard deviation for normally distributed otherwise median [interquartile range, $\mathrm{M}(\mathrm{QR})]$. The non-normal distributed FeNO values were $\log$ transformed. The comparisons between groups were used one-way analysis of variance. The diagnostic value of the FeNO for predicting BHR was revealed by ROC curve in AR patients. $\mathrm{P}<0.05$ was considered to indicate a statistically significant difference.

\section{Results}

Demographic and baseline characteristics. A total of 102 subjects underwent screening, 93 subjects (45 AR patients, 20 AR with asthma patients, and 28 normal controls) enrolled in this study and included in the analysis. The AR group was divided into two sub-groups: AR with asymptomatic BHR group and the AR without BHR group. There were no significant differences in baseline demographic characteristics (Table I). The spirometric parameters (FEV 1 , PEF, MMEF, $\mathrm{MEF}_{50 \%}$ and $\mathrm{MEF}_{25 \%}$ percent predicted) in the AR with asthma group were lower compared with that in AR and normal control groups with significant differences $(\mathrm{P}<0.05)$, except $\mathrm{FVC}$ percent predicted and $\mathrm{FEV}_{1} / \mathrm{FVC} \%$ ( $\mathrm{P}>0.05$ ) (Table I). The duration periods of AR and asthma were 7.5 (9.0) and 5.0 (8.0) years, respectively, in the AR with asthma group; and the duration period of AR was 9.0 (4.5) years in AR group, where the values in brackets 
Table I. Demographic and baseline characteristics.

\begin{tabular}{|c|c|c|c|c|}
\hline Characteristic & $\operatorname{AR}(n=45)$ & AR and asthma $(n=20)$ & Control (n=28) & P-value \\
\hline Age (year) & $27.9 \pm 9.8$ & $33.6 \pm 10.1$ & $30.8 \pm 12.1$ & $>0.05$ \\
\hline Gender (male) & 18 & 10 & 16 & $>0.05$ \\
\hline Height (cm) & $163.4 \pm 8.3$ & $162.7 \pm 9.1$ & $164.1 \pm 8.2$ & $>0.05$ \\
\hline Weight (kg) & $56.6 \pm 9.7$ & $53.9 \pm 7.0$ & $58.4 \pm 8.6$ & $>0.05$ \\
\hline FVC pred (\%) & $97.15 \pm 11.78$ & $89.04 \pm 9.48$ & $99.68 \pm 13.17$ & $>0.05$ \\
\hline $\mathrm{FEV}_{1}$ pred $(\%)$ & $94.56 \pm 10.83$ & $79.07 \pm 8.53$ & $94.97 \pm 9.17$ & $<0.05$ \\
\hline $\mathrm{FEV}_{1} / \mathrm{FVC}(\%)$ & $83.15 \pm 4.87$ & $77.35 \pm 11.49$ & $80.70 \pm 6.25$ & $>0.05$ \\
\hline PEF pred $(\%)$ & $99.48 \pm 12.39$ & $83.70 \pm 14.01$ & $108.61 \pm 10.96$ & $<0.05$ \\
\hline MMEF pred (\%) & $76.85 \pm 14.47$ & $49.88 \pm 15.62$ & $73.77 \pm 18.86$ & $<0.05$ \\
\hline $\mathrm{MEF}_{50 \%}$ pred (\%) & $80.19 \pm 16.67$ & $51.67 \pm 14.35$ & $78.95 \pm 18.87$ & $<0.05$ \\
\hline $\mathrm{MEF}_{25 \%}$ pred $(\%)$ & $73.72 \pm 17.23$ & $48.90 \pm 18.71$ & $66.78 \pm 20.88$ & $<0.05$ \\
\hline FeNO (ppb) & $29.5(22.0)$ & $61.5(33.0)$ & $16.0(10.0)$ & $<0.05$ \\
\hline EOs in sputum (\%) & $4.36 \pm 4.76$ & $18.58 \pm 11.26$ & $0.45 \pm 0.73$ & $<0.05$ \\
\hline
\end{tabular}

AR, allergic rhinitis; FVC, forced vital capacity; pred, predicted; FEV, forced expiratory volume in 1 sec; PEF, peak expiratory flow; MMEF, maximal (mid-)expiratory flow; $\mathrm{MEF}_{50 / 25 \%}$, maximal expiratory flow at 25/50\%; FeNO, fractional exhaled nitric oxide; EOs, eosinophils.

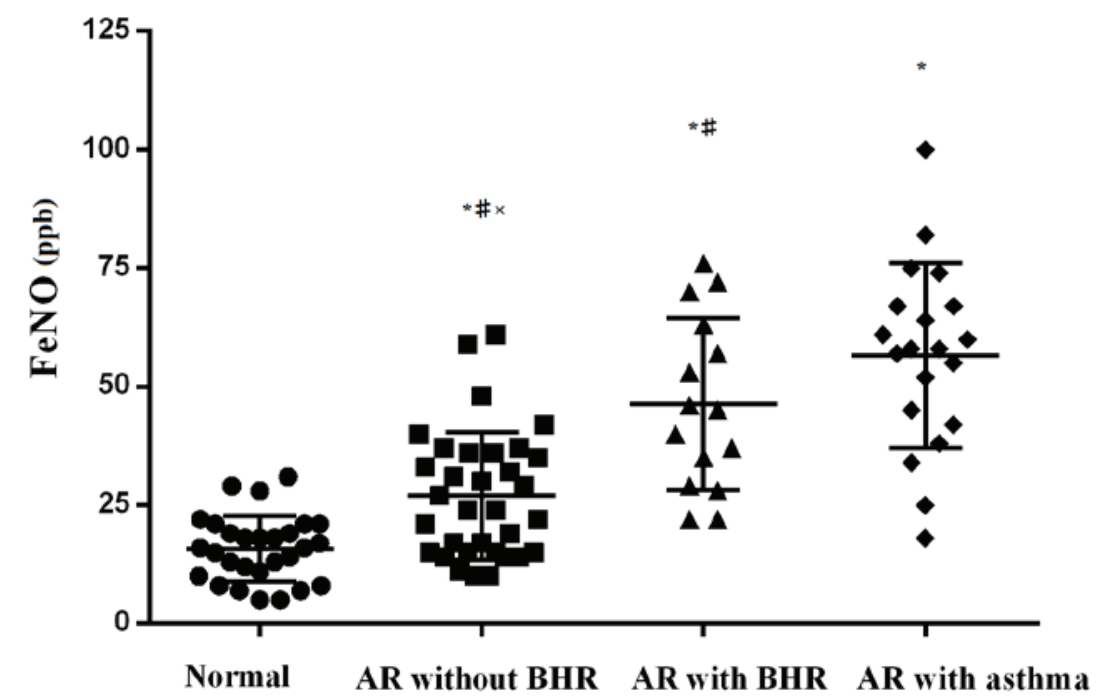

Figure 2. Distributions of FeNO in different groups. ${ }^{*} \mathrm{P}<0.05$, compared with the control group; ${ }^{\text {}} \mathrm{P}<0.05$ compared with the AR + asthma group; ${ }^{\times} \mathrm{P}<0.05$ compared between AR with BHR and the AR without BHR groups. FeNO, fractional exhalation of nitric oxide. FeNO, fractional exhaled nitric oxide; AR, allergic rhinitis; BHR, bronchial hyperresponsiveness.

show the interquartile range of the duration of the disease. The severities of AR in the AR with asthma groups were predominantly moderate to severe.

FeNO levels and diagnostic sensitivity/specificity. The value of FeNO was significantly higher in the AR with asthma group compared with the AR group, and higher in the AR group compared with the control group [61.5 (33.0) vs. 29.5 (22.0) vs. $16.0(10.0) \mathrm{ppb} ; \mathrm{P}<0.05]$. The values in bracket indicate the interquartile range. The distribution (including the two sub-groups of AR) of FeNO is shown in Fig. 2. An increasing trend was observed in the value of FeNO from normal controls to $\mathrm{AR}$ without BHR, AR + BHR and AR + asthma groups.
Patients with abnormal FeNO values (>25.0 ppb) were significantly more in the AR with asthma group compared with the other three groups and subgroups $(\mathrm{P}<0.05)$. As shown in Fig. 3, receiver operating characteristic curves (ROC) revealed a moderately increased screening capability of FeNO for the diagnosis of BHR/asthma (AUC, 0.910; 95\%CI, 0.836-0.984) compared with for asthma only (AUC, 0.873; 95\% CI, 0.753-0.992). Furthermore, when taking the cut-off values at 31.5 and $38.0 \mathrm{ppb}$, the sensitivity and specificity values were $0.750,0.737$ and $0.750,0.831$, respectively.

Eosinophil count. The percentages of eosinophils in induced sputum were $0.45 \pm 0.73,4.36 \pm 4.76$ and $18.58 \pm 11.26 \%$ in the 


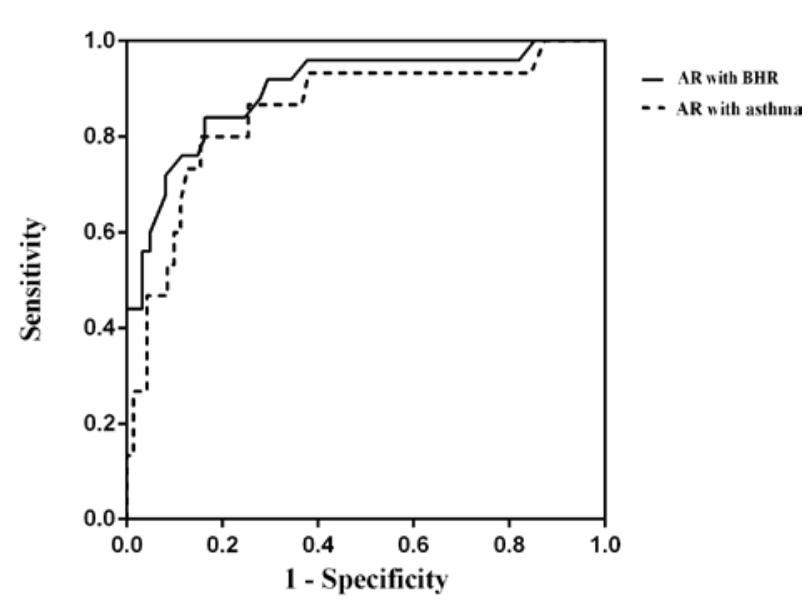

Figure 3. Receiver operating characteristic curves of fractional exhaled nitric oxide for the diagnosis of BHR or asthma in allergic rhinitis. BHR, bronchial hyperresponsiveness.

control, AR and AR with asthma groups, respectively $(\mathrm{P}<0.05)$. The value of FeNO and the percentages of eosinophils in induced sputum correlate well $(\mathrm{r}=0.70, \mathrm{P}<0.01)$. There is a similar trend of the percentage of eosinophils in sputum from normal controls to AR without BHR, AR + BHR and AR with asthma groups (Fig. 4). The AR without BHR exhibited a significantly higher mean percentage of eosinophils compared with the AR with BHR group (2.43 \pm 3.57 vs. $7.35 \pm 4.98 \%$; $\mathrm{P}=0.047)$.

\section{Discussion}

In the present study, the values of FeNO in the normal control group were substantially reduced compared with the AR with asthma groups, which was consistent with the count of eosinophils in the sputum, suggesting that the value of FeNO may be used as an indicator of eosinophil inflammation in lower airways. The high diagnostic value of FeNO in predicting BHR or asthma in AR patients was revealed by the ROC curve. When taking the cut-off value to be $31.5 \mathrm{ppb}$, the sensitivity and specificity were 0.846 and 0.817 for diagnosing BHR, while the sensitivity and specificity were 0.857 and 0.847 for the diagnosis of asthma (cut-off value, $38.0 \mathrm{ppb}$ ). A prior study demonstrated that the eosinophil inflammation in the lower airways may be correlated with BHR (13), therefore it is possible that the measurement of FeNO may predict BHR in AR patients with or without asthma, and the present results were consistent with previous findings $(11,20)$. Furthermore, the measurement of FeNO is non-invasive, simple, reproducible and safe. Thus we propose that the measurement of FeNO may be used for the early detection of inflammation and BHR in AR patients.

In the present study, the median value of FeNO [29.5 (22.0) ppb] in the AR group was higher compared with the mean value [17.7 (6.1) ppb] of Guangzhou normal adults, where the values in bracket are the interquartile range. In addition, the FeNO values of $10 \%$ (3/30) of AR without BHR subjects were higher than the upper limit of normal values (ULN; $41.0 \mathrm{ppb)}$ of Guangzhou adults, which suggests that even in AR patients without BHR or lower airway symptoms there may already detected lower airways inflammation. The

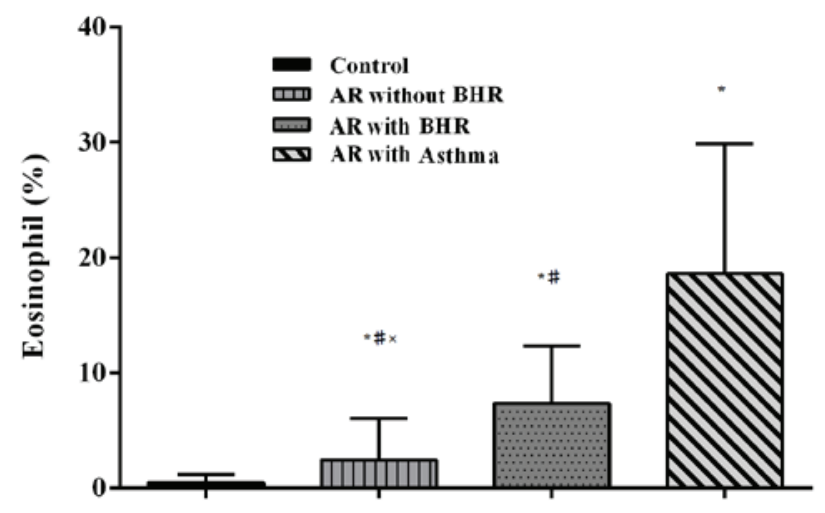

Figure 4. Eosinophils in induced sputum of different groups. ${ }^{*} \mathrm{P}<0.05$ compared with the control group; ${ }^{\#} \mathrm{P}<0.05$ compared with the $\mathrm{AR}+$ asthma group; ${ }^{\times} \mathrm{P}<0.05$ compared between $\mathrm{AR}$ with $\mathrm{BHR}$ and $\mathrm{AR}$ without the BHR groups. AR, allergic rhinitis; BHR, bronchial hyperresponsiveness.

percentages of subjects with higher FeNO values than the ULN values of Guangzhou adults in AR with asymptomatic BHR and AR + asthma groups were 60.0 and $65 \%$, increasingly.

As is an independent risk factor of asthma, AR promotes the development and affects the control of asthma. It has been demonstrated that moderate/severe persistent AR was a higher risk factor than seasonal and mild persistent AR for AR patients developing asthma (21). A proportion of AR patients experienced AR, AR with asymptomatic BHR, then with lower airways symptoms and finally developed to asthma. There is a consecutive process in the progression of AR into asthma, as previously demonstrated by a long-term follow-up study (22). This has also been confirmed through a retrospectively study $(23,24)$. The present results showed that the mean duration of AR was longer than asthma in the AR with asthma group, although certain patients reported that asthma was diagnosed earlier than AR. Although there are different phenotypes of asthma, and not all asthma phenotypes are characterized by elevated FeNO levels, the measurement of FeNO remained useful for detecting BHR in AR, as the majority of atopic asthmatics exhibited elevated eosinophilic inflammation and FeNO levels. The present findings demonstrated the use of FeNO in detecting the consecutive progression of AR to AR with BHR, and ultimately to AR with asthma, which supports the hypothesis that AR and asthma represent 'one airway, one disease'. The early diagnosis of BHR in AR and resulting early intervention may help to prevent the development of asthma and other complications from AR.

Several limitations of the study were considered. Firstly, the present study was a cross-section survey, which can not directly demonstrate the process of AR developed to asthma. Secondly, the sample size was limited in each group, although they were unselected subjects. Thirdly, not all AR patients ultimately developed asthma, so prospective studies with duration and severity classification of AR patients are required.

In conclusion, the present results suggest that FeNO measurement, as a simple and effective method of evaluation lower airway inflammation, may be employed for predicting $\mathrm{BHR} / \mathrm{asthma}$ in patients with AR, and supervising the consecutive process. 


\section{Acknowledgements}

This study was supported by Changjiang Scholars and Innovative Research Team in University (grant no. ITR0961); The National Key Technology R\&D Program of the 12th National Five-year Development Plan (grant no. 2012BAI05B00); The National Natural Science Foundation of China (grant no. 81300017). The authors thank Qingxia Liu (Qingyuan People's Hospital), Guo E (Xiangyang Central Hospital), Wenhua Jian (First Affiliated Hospital of Guangzhou Medical University), Zhiyu Liang (Yuexiu People's Hospital), Linting Luo, Diteng Luo, Xiangong Xu, Huayi Huang, Yongqing Ye and Xianmiao Ye (all from the Guangzhou Medical University) for their assistance in participant recruitment.

\section{References}

1. Zhang Y and Zhang L: Prevalence of allergic rhinitis in China. Allergy Asthma Immunol Res 6: 105-113, 2014.

2. Grossman J: One airway, one disease. CHEST 111 (2 Suppl): 11S-16S, 1997.

3. Lin J, Su N, Liu G, Yin K, Zhou X, Shen H, Chen P, Chen R, Liu C, Wu C, et al: The impact of concomitant allergic rhinitis on asthma control: A cross-sectional nationwide survey in China. J Asthma 51: 34-43, 2014.

4. Bonay M, Neukirch C, Grandsaigne M, Leçon-Malas V, Ravaud P, Dehoux M and Aubier M: Changes in airway inflammation following nasal allergic challenge in patients with seasonal rhinitis. Allergy 61: 111-118, 2006.

5. Linneberg A,Henrik Nielsen N,Frølund L, Madsen F, Dirksen A and Jørgensen T; Copenhagen Allergy Study: The link between allergic rhinitis and allergic asthma: A prospective population-based study. The copenhagen allergy study. Allergy 57: 1048-1052, 2002.

6. Brozek JL, Bousquet J, Baena-Cagnani CE, et al: Allergic Rhinitis and its Impact on Asthma (ARIA) guidelines: 2010 revision. J Allergy Clin Immunol. 126: 466-476, $2010 .$.

7. Alvarez MJ, Olaguibel JM, Garcia BE, Tabar AI and Urbiola E: Comparison of allergen-induced changes in bronchial hyperresponsiveness and airway inflammation between mildly allergic asthma patients and allergic rhinitis patients. Allergy 55: 531-539, 2000.

8. Irvin CG: The nose: a window into the asthmatic lung? Clin Exp Allergy 40: 839-840, 2010.

9. Bhimrao SK, Wilson SJ and Howarth PH: Airway inflammation in atopic patients: a comparison of the upper and lower airways. Otolaryngol Head Neck Surg 145: 396-400, 2011.

10. Braunstahl GJ, Fokkens WJ, Overbeek SE, KleinJan A, Hoogsteden HC and Prins JB: Mucosal and systemic inflammatory changes in allergic rhinitis and asthma: a comparison between upper and lower airways. Clin Exp Allergy 33: 579-587, 2003.
11. Smith AD, Cowan JO, Filsell S, McLachlan C, Monti-Sheehan G, Jackson P and Taylor DR: Diagnosing asthma: comparisons between exhaled nitric oxide measurements and conventional tests. Am J Respir Crit Care Med 169: 473-478, 2004.

12. Wang DY, Goh DY, Ho AK, Chew FT, Yeoh KH and Lee BW: The upper and lower airway responses to nasal challenge with house-dust mite Blomia tropicalis. Allergy 58: 78-82, 2003.

13. de Graaf-int Veld C, Garrelds IM, Koenders S and Gerth van Wijk R: Relationship between nasal hyperreactivity, mediators and eosinophils in patients with perennial allergic rhinitis and controls. Clin Exp Allergy 26: 903-908, 1996.

14. Global Strategy for Asthma Management and Prevention. Global Initiative for Asthma (GINA) 2011. http://www.ginasthma.org. Accessed December 20, 2011.

15. Luo W, Lai KF, Chen RC, Chen QL and Zhong NS: Establishment of reference values for cellularity in induced sputum of healthy adults in Guangzhou. Guo Ji Hu Xi Za Zhi She 16: 1213-1215, 2007 (In Chinese).

16. American Thoracic Society; European Respiratory Society: ATS/ERS recommendations for standardized procedures for the online and offline measurement of exhaled lower respiratory nitric oxide and nasal nitric oxide, 2005. Am J Respir Crit Care Med 171: 912-930, 2005.

17. Jiang CY, Zheng JP, Guan WJ, Xie YQ, Gao Y, Liu QX, An JY, Yu XX, Liu WT: Reference ranges and predicted equations for fractional exhaled nitric oxide in adult hans. Zhong Guo Shi Yong Nei Ke Za Zhi 8: 608-613, 2012 (In Chinese).

18. Miller MR, Hankinson J, Brusasco V, Burgos F, Casaburi R, Coates A, Crapo R, Enright P, van der Grinten CP, Gustafsson P, Jensen R, Johnson DC, MacIntyre N, McKay R, Navajas D, Pedersen OF, Pellegrino R, Viegi G, Wanger J; ATS/ERS Task Force: Standardization of spirometry. Eur Respir J 26: 319-338, 2005.

19. Zheng JP and Zhong NS: Normative values of pulmonary function testing in Chinese adults. Chin Med J (Engl) 115: 50-54, 2002.

20. Schneider A, Schwarzbach J, Faderl B, Welker L, Karsch-Völk M, Jörres RA: FENO measurement and sputum analysis for diagnosing asthma in clinical practice. Respir Med 107: 209-216, 2013.

21. Marcucci F, Passalacqua G, Canonica GW, Frati F, Salvatori S, Di cara G, Petrini I, Bernini M, Novembre E, Bernardini R, et al: Lower airway inflammation before and after house dust mite nasal challenge: An age and allergen exposure-related phenomenon. Respir Med 101: 1600-1608, 2007.

22. Settipane RJ, Hagy GW and Settipane GA: Long-term risk factors for developing asthma and allergic rhinitis: a 23-year follow-up study of college students. Allergy Proc 15: 21-25, 1994.

23. Stachler RJ: Comorbidities of asthma and the unified airway. Int Forum Allergy Rhinol 5: S17-S22, 2015.

24. Moshe S, Slodownik D, Yagev Y, Segal N, Tavor M, Afek A and Zack O: Atopy as a risk factor for the development of asthma in young recruits. J Asthma 52: 453-457, 2015. 